\title{
Clinicopathologic Features Predicting Involvement of Non- sentinel Axillary Lymph Nodes in Iranian Women with Breast Cancer
}

\author{
Seyed Alireza Moosavi ${ }^{1}$, Afshin Abdirad ${ }^{2}$, Ramesh Omranipour ${ }^{1}$, Maryam Hadji ${ }^{3}$, \\ Amirnader Emami Razavi ${ }^{4}$, Massoome Najafi*
}

\begin{abstract}
Background: Almost half of the breast cancer patients with positive sentinel lymph nodes have no additional disease in the remaining axillary lymph nodes. This group of patients do not benefit from complete axillary lymph node dissection. This study was designed to assess the clinicopathologic factors that predict non-sentinel lymph node metastasis in Iranian breast cancer patients with positive sentinel lymph nodes. Materials and Methods: The records of patients who underwent sentinel lymph node biopsy, between 2003 and 2012, were reviewed. Patients with at least one positive sentinel lymph node who underwent completion axillary lymph node dissection were enrolled in the present study. Demographic and clinicopathologic characteristics including age, primary tumor size, histological and nuclear grade, lymphovascular invasion, perineural invasion, extracapsular invasion, and number of harvested lymph nodes, were evaluated. Results: The data of 167 patients were analyzed. A total of $92(55.1 \%)$ had non-sentinel lymph node metastasis. Univariate analysis of data revealed that age, primary tumor size, histological grade, lymphovascular invasion, perineural invasion, extracapsular invasion, and the number of positive sentinel lymph nodes to the total number of harvested sentinel lymph nodes ratio, were associated with non-sentinel lymph node metastasis. After logistic regression analysis, age $(\mathrm{OR}=0.13 ; 95 \% \mathrm{CI}$, 0.02-0.8), primary tumor size (OR=7.7; 95\% CI, 1.4-42.2), lymphovascular invasion $(O R=19.4 ; 95 \%$ CI, 1.4268.6), extracapsular invasion $(\mathrm{OR}=13.3 ; 95 \% \mathrm{CI}, 2.3-76)$, and the number of positive sentinel lymph nodes to the total number of harvested sentinel lymph nodes ratio $(\mathrm{OR}=20.2 ; 95 \% \mathrm{CI}, 3.4-121.9)$, were significantly associated with non-sentinel lymph node metastasis. Conclusions: According to this study, age, primary tumor size, lymphovascular invasion, extracapsular invasion, and the ratio of positive sentinel lymph nodes to the total number of harvested sentinel lymph nodes, were found to be independent predictors of non-sentinel lymph node metastasis.
\end{abstract}

Keywords: Breast cancer - sentinel node biopsy - non-sentinel node metastasis - predictors

Asian Pac J Cancer Prev, 15 (17), 7049-7054

\section{Introduction}

Axillary lymph node (ALN) status is an important factor in the staging, prognosis and selection of an appropriate treatment modality in early breast cancer. Axillary dissection is currently the standard of care in patients with a positive sentinel lymph node (SLN) (Mcmasters et al., 2000; Cady, 2001). Recently, the survival benefit of completion axillary lymph node dissection (ALND) for all patients with a positive SLN has been questioned (Giuliano et al., 2011). Some studies have indicated that non-sentinel lymph node (NSLN) metastasis was observed in only $35 \%$ to $50 \%$ of breast cancer patients with a positive SLN (Chu et al., 1999; Turner et al., 2000). Therefore, $50 \%$ to $65 \%$ of patients with a positive SLN suffer from the morbidity of unnecessary ALND, such as hand paresthesia, shoulder dysfunction and lymphedema (Schrenk et al., 2000; Lucci et al., 2007; Ashikaga et al., 2010). Nowadays, there is an increased tendency to avoid completion ALND in selected patients with a positive SLN (Noguchi, 2008).

Many studies have identified factors including tumor size, histological type, nuclear and histological grade, lymphovascular invasion (LVI), estrogen and progesterone receptor (ER and PR) status, and HER-2/neu expression, as predictors of NSLN metastasis (NSLNM) in patients with a positive SLN (Yu et al., 2005; Ozmen et al., 2006; Wada et al., 2006; Kapur et al., 2007; Boler et al., 2012; Eldweny et al., 2012). These factors have been used to develop nomograms to predict the risk of NSLNM (Van Zee et al., 2003; Barranger et al., 2005; Kohrt et al., 2008; Pal et al., 2008). The validity and accuracy of

\footnotetext{
${ }^{1,3}$ Department of Surgery, ${ }^{2}$ Department of Pathology, ${ }^{3}$ Cancer Research Center, ${ }^{4}$ Iran National Tumor Bank, Tehran University of
} Medical Sciences, Tehran, Iran*For correspondence: mdmasoume@yahoo.com 
clinicopathologic factors to predict NSLNM were different in various studies. A meta-analysis performed by Van la Parra et al. in the Netherlands, analyzed the results of 56 studies on predictive factors published between January 1999 and June 2009. Among all the variables which had a significant association with NSLNM in individual studies, only eight characteristics were found to have the highest likelihood to predict NSLNM (Van La Parra et al., 2011).

In spite of the numerous studies, it is still not yet clear whether a subgroup of patients with a positive SLN can be safely spared completion ALND. The purpose of this study was to define the clinicopathologic features of the primary tumor and SLN associated with NSLNM in patients with a positive SLN in a cancer referral center in Iran.

\section{Materials and Methods}

We reviewed the medical records and pathology reports of patients who had undergone a SLN biopsy in Cancer Institute, Tehran University of Medical Sciences, between 2003 and 2012. The patients who had at least one positive SLN and underwent completion ALND were enrolled in the present study. The inclusion criteria were the presence of micro or macrometastasis, or isolated tumor cells (ITCs) in the SLN. Patients who received neo-adjuvant chemotherapy were excluded from the study.

SLN biopsies were performed using blue dye method, radiocolloide injection, or a combination of both methods, by surgeons trained for SLNB. The detection methods of SLN metastasis were frozen sectioning during the operation and standard staining of paraffin sections.

Primary tumor size was classified as T1 $(\leq 20 \mathrm{~mm}), \mathrm{T} 2$ $(20<$ size $\leq 50 \mathrm{~mm})$, and T3 $(>50 \mathrm{~mm})$ (Singletary et al., 2002). The size of the SLN metastasis was categorized according to the American Joint Committee on Cancer (AJCC) in the sixth edition of the Cancer Staging Manual. Lymph node metastatic lesions with a maximum diameter of $\geq 2 \mathrm{~mm}$ were defined as macrometastasis ( $\mathrm{pN} 1$ ), lesions with a diameter of $0.2-2 \mathrm{~mm}$ as micrometastasis ( $\mathrm{pNmi}$ ), and a lesion of single tumor cells, or small cell clusters with a diameter $<0.2 \mathrm{~mm}$ were defined as ITCs [pNO(i+)] (Singletary and Greene, 2003). Histological and nuclear grade based on a modified Scarff-Bloom \& Richardson score were divided into three grades.

Pathology reports and the original hematoxylin and eosin (H\&E) slides were reviewed for histological size

Table 1. Univariate and Multivariate Analysis of Association of Clinicopathological Characteristics with Nonsentinel Lymph Node Metastasis.

\begin{tabular}{|c|c|c|c|c|c|c|c|}
\hline \multirow[t]{2}{*}{ Variable } & & \multicolumn{3}{|c|}{ Non sentinel lymph node } & \multicolumn{3}{|c|}{ Odds Ratio $(95 \% \mathrm{CI})$} \\
\hline & & $\begin{array}{c}\text { Negative } \\
\text { NSLN(75) }\end{array}$ & $\begin{array}{c}\text { Positive } \\
\text { NSLN(92) }\end{array}$ & P-Value & Crude OR & Adjusted OR & P-value \\
\hline \multirow[t]{2}{*}{ Age } & $<40$ & $14(35.9 \%)$ & $25(64.1 \%)$ & 0.001 & Reference & Reference & \\
\hline & $\geq 40$ & $61(48.4 \%)$ & $65(51.6 \%)$ & & $0.6(0.3-1.3)$ & $0.13(0.02-0.8)$ & 0.03 \\
\hline \multirow[t]{3}{*}{ Histological tumor size (mm) } & $\mathrm{T} 1($ size $\leq 20)$ & $26(72.2 \%)$ & $10(27.8 \%)$ & & Reference & Reference & \\
\hline & $\mathrm{T} 2(20<$ size $\leq 50)$ & $47(40.9 \%)$ & $68(59.1 \%)$ & 0.001 & $3.1(1.5-6.6)$ & $7.7(1.4-42.2)$ & 0.02 \\
\hline & $\mathrm{T} 3($ size $>50)$ & - & $11(100 \%)$ & & --- & --- & \\
\hline \multirow[t]{2}{*}{ Multifocality } & NO & $67(49.2 \%)$ & $69(50.8 \%)$ & 0.06 & Reference & Reference & 0.2 \\
\hline & YES & $8(29.6 \%)$ & $19(70.4 \%)$ & & $1.9(0.8-4.9)$ & $0.2(0.02-1.9)$ & \\
\hline \multirow[t]{4}{*}{ Tumor histology } & Ductal & $68(44.7 \%)$ & $84(55.3 \%)$ & 0.4 & --- & --- & --- \\
\hline & Lobular & $5(55.5 \%)$ & $4(44.5 \%)$ & & --- & --- & --- \\
\hline & Mixed & $1(25 \%)$ & $4(75 \%)$ & & --- & --- & --- \\
\hline & other & $1(1.3 \%)$ & --- & & 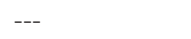 & --- & --- \\
\hline \multirow[t]{3}{*}{ Nuclear grade } & Grade 1 & $8(57.1 \%)$ & $6(42.9 \%)$ & 0.2 & Reference & Reference & \\
\hline & Grade 2 & $51(45.9 \%)$ & $60(54.1 \%)$ & & $1.1(0.3-3.7)$ & $0.3(0.02-2.9)$ & 0.3 \\
\hline & Grade 3 & $9(30 \%)$ & $21(70 \%)$ & & $2.1(0.5-8.4)$ & $0.1(0.004-1.2)$ & 0.1 \\
\hline \multirow[t]{3}{*}{ Histological grade } & Grade 1 & $15(65.2 \%)$ & $8(34.8 \%)$ & 0.02 & Reference & Reference & \\
\hline & Grade 2 & $46(42.6 \%)$ & $62(57.4 \%)$ & & $2(0.7-5.2)$ & $2.1(0.4-11.4)$ & 0.4 \\
\hline & Grade 3 & $6(26 \%)$ & $17(74 \%)$ & & $3.8(1.01-13.8)$ & $5.5(0.3-91.8)$ & 0.2 \\
\hline \multirow[t]{2}{*}{ Estrogen Receptor } & NO & $14(42.4 \%)$ & $19(57.6 \%)$ & 0.7 & --- & --- & --- \\
\hline & YES & $53(45.7 \%)$ & $63(54.3 \%)$ & & 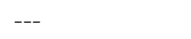 & --- - & --- \\
\hline \multirow[t]{2}{*}{ Progesterone Receptor } & NO & $17(37 \%)$ & $29(63 \%)$ & 0.2 & Reference & Reference & \\
\hline & YES & $50(48.5 \%)$ & $53(51.5 \%)$ & & $0.6(0.3-1.2)$ & $1.7(0.3-9.6)$ & 0.6 \\
\hline \multirow[t]{2}{*}{ Her-2/ neu } & NO & $44(42.7 \%)$ & $59(57.3 \%)$ & 0.3 & --- & --- & --- \\
\hline & YES & $23(51 \%)$ & $22(49 \%)$ & & --- & --- & --- \\
\hline \multirow[t]{2}{*}{ P53 } & NO & $23(36.5 \%)$ & $40(63.5 \%)$ & 0.2 & Reference & Reference & \\
\hline & YES & $23(47 \%)$ & $26(53 \%)$ & & $0.7(0.3-1.4)$ & $0.7(0.2-3)$ & 0.9 \\
\hline \multirow[t]{2}{*}{ Lymphovascular invasion } & NO & $22(78.6 \%)$ & $6(21.4 \%)$ & 0.001 & Reference & Reference & \\
\hline & YES & $51(38 \%)$ & $83(62 \%)$ & & $4.8(1.8-13)$ & $19.4(1.4-268.6)$ & 0.03 \\
\hline \multirow[t]{2}{*}{ Preineural invasion } & NO & $53(56.4 \%)$ & $41(43.6 \%)$ & 0.001 & Reference & Reference & \\
\hline & YSE & $18(29.5 \%)$ & $43(70.5 \%)$ & & $2.5(1.2-5)$ & $0.8(0.2-3.4)$ & 0.7 \\
\hline \multirow[t]{2}{*}{ Type of diagnosis } & Frozen & $62(43.3 \%)$ & $81(56.7 \%)$ & 0.4 & --- & 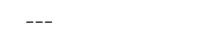 & --- \\
\hline & Standard paraffin & $11(52.4 \%)$ & $10(47.6 \%)$ & & --- & --- & --- \\
\hline \multirow[t]{2}{*}{ Size of SLN metastasis } & Macrometastasis & $61(42 \%)$ & $84(58 \%)$ & 0.1 & Reference & Reference & \\
\hline & Micrometastasis & $9(64.3 \%)$ & $5(35.7 \%)$ & & $0.4(0.1-1.3)$ & $1.7(0.1-20.7)$ & 0.7 \\
\hline \multirow[t]{2}{*}{ Extracapsular invasion } & NO & $51(57.3 \%)$ & $38(42.7 \%)$ & 0.001 & Reference & Reference & \\
\hline & YES & $15(25.4 \%)$ & $44(74.6 \%)$ & & $3.1(1.5-6.6)$ & $13.3(2.3-76)$ & 0.004 \\
\hline \multirow[t]{2}{*}{ Number of positive SLN } & 1 & $54(47.4 \%)$ & $60(52.6 \%)$ & 0.3 & --- & --- & --- \\
\hline & $>1$ & $21(39.6 \%)$ & $32(0.6 \%)$ & & --- & --- & --- \\
\hline \multirow[t]{2}{*}{ PSLN/TSLN ratio } & $<100 \%$ & $49(62.8 \%)$ & $29(37.2 \%)$ & 0.0001 & Reference & Reference & \\
\hline & $100 \%$ & $26(29.2 \%)$ & $63(70.8 \%)$ & & $4.1(2.1-7.8)$ & $20.2(3.4-121.9)$ & 0.001 \\
\hline
\end{tabular}


and multifocality of the primary tumor,LVI and perineural invasion (PNI) in the area of the primary tumor, nuclear and histological grade, histological type of tumor, detection method of SLN metastasis, size of SLN metastasis (micro or macrometastasis), extracapsular invasion (ECI) in the SLN, number of harvested and positive SLNs, and NSLNs. The ER, PR and P53 status, and HER-2/neu expression were extracted from the patients' medical records.

We studied the association of NSLNM, as an outcome in patients with positive SLN, with age, histological size, multifocality, histological type of the primary tumor, LVI, PNI, ER, PR and P53 status, HER2/neu expression, nuclear grade, histological grade, detection method of SLN metastasis, ECI, number of positive SLNs, number of positive SLNs to the total number of harvested SLNs (PSLNs/TSLNs) ratio, and the size of the SLN metastasis. We used a logistic regression model to estimate odds ratio (OR) and corresponding 95\% confidence interval (95\% $\mathrm{CI})$. Results of the crude and adjusted regression model were presented. Factors significantly related to NSLNM in patients with a positive SLN with a p-value of 0.2 or less were entered into a backward stepwise multiple logistic regression model. We carried out a Co-linearity test between variables to control co-variability between the variables and thus identify independent predictors for the NSLNM in patients with a positive SLN. In addition, we excluded variables with a p-value of more than 0.2 from the model, although we presented the crude ORs for all putative risk factors. We used Stata statistical software (version 11) to perform the statistical analyses. The Regional Ethics Committee of Tehran University of Medical Sciences approved this study.

\section{Results}

The files and pathology reports of 607 patients who underwent a SLN biopsy between 2003 and 2012 were reviewed. Data of 167 female breast cancer patients who had a positive SLN on frozen or permanent pathology were analyzed. The mean age of the patients were $47.4( \pm 10.7)$ years. The average number of harvested SLNs was 2.3 $( \pm 1.4)$ and the average number of positive SLNs was 1.5 $( \pm 0.95)$. Ninety two patients $(55.1 \%)$ had NSLNM. The average number of harvested NSLNs was $9.9( \pm 4.1)$ and the average number of positive NSLNs was $2.3( \pm 3.2)$.

Univariate analysis revealed that age, histological tumor size, multifocality, nuclear grade, histological grade, PR and P53 status, LVI, PNI, size of SLN metastasis, ECI and PSLNs/TSLNs ratio, were significantly associated with NSLNM in patients with positive SLN (Table 1). However, in the multivariate logistic regression age, LVI, ECI, primary tumor size, and PSLNs/TSLNs ratio, remained significant predictors of NSLNM. Patients who were over 40 years had an $87 \%$ lower risk of NSLNM compared to those who were younger than 40 years $(\mathrm{OR}=0.13 ; 95 \% \mathrm{CI}, 0.02-0.8)$. The risk of NSLNM was19fold higher in patients with LVI $(\mathrm{OR}=19.4$; 95\% CI, 1.4268.6). In addition, patients with ECI had a 13-fold higher risk of NSLNM compared to those without $\mathrm{ECI}(\mathrm{OR}=13.3$; 95\% CI, 2.3-76). Moreover, patients with histological size of the primary tumor between 20 and $50 \mathrm{~mm}$ had a higher risk of NSLNM compared to patients with a tumor size less than $20 \mathrm{~mm}(\mathrm{OR}=7.7$; 95\% CI, 1.4-42.2). The risk of NSLNM in patients with a PSLNs/TSLNs ratio of $100 \%$ was 20-fold higher compared to patients with a ratio of less than $100 \%(\mathrm{OR}=20.2$; 95\% CI, 3.4-121.9) (Table 1).

\section{Discussion}

Recently, the role of ALND as a standard of care in patients with positive SLN has been questioned. Almost $50 \%$ of breast cancer patients with a positive SLN who undergo ALND have no additional disease in NSLNs and this subset of patients do not benefit from this intervention. There are also some reports of the low incidence of regional failure in patients with SLN metastasis who did not undergo ALND because of associated comorbidity or patient refusal (Fant et al., 2003; Guenther et al., 2003; Jeruss et al., 2005).

Based on these observations, numerous studies have been performed to determine the predictive factors of NSLN involvement in patients with SLN metastasis in order to identify a subset of patients who can be spared a negative ALND safely.

The relationship of age to the prognosis of breast cancer is confirmed in different studies. Breast cancer in younger patients appears to be more aggressive (Dubsky et al., 2002; Afsharfard et al., 2013). Some studies in Iran have suggested that the age of Iranian women with breast cancer is at least one decade younger, in comparison with developed countries (Harirchi et al., 2004; Mousavi et al., 2007). According to these studies, the patients in our study were divided into two groups: younger than 40 years and older than 40 years. Studies have revealed that there is an inverse correlation between age and the involvement of axillary nodes (Aitken and Osman, 2010). In this study, patients' age was an independent predictor of NSLNM (OR=0.13; 95\% CI, 0.02-0.8). The effect of age on NSLNM has been evaluated in many other studies. However, we found only one study in which age was a predictor of NSLNM (Farshid et al., 2004), and in the other studies, no relationship was found between age and NSLNM.

Multivariate analysis indicated a significant association between PSLNs/TSLNs ratio and NSLNM. This ratio was the strongest predictor of NSLNM in this study. Patients with PSLNs/TSLNs ratio of $100 \%$ had a higher likelihood of NSLNM. This finding indicates that patients with at least one negative SLN have a lower risk of NSLNM compared to those with involvement of all SLNs. Similar findings have been reported by Goyal et al. (Goyal et al., 2004). They mentioned that a greater number of negative SLNs indicated a lower lymphatic tumor burden and decreased likelihood of NSLNM.

LVI and ECI were the other two significant predictors of NSLNM in this study with ORs of 19.4 and 13.3 respectively. LVI as a predictor of NSLNM has been reported in several studies (Silverstein et al., 2001; Viale et al., 2005; Bolster et al., 2007; Jinno et al., 2008; Fougo et al., 2009; Alvarenga et al., 2013). LVI, overall metastasis size and PSLNs/TSLNs ratio, were three predicting factors of NSLNM which were reported by Gur et al. (Gur et al., 
2010). In their study, the highest OR belonged to LVI. ECI of the axillary lymph node is an index for aggressive tumor behavior in breast cancer and the patients with ECI have an obvious poorer outcome (Altinyollar et al., 2007). In many studies, ECI was a predictor of NSLNM (Stitzenberg et al., 2003; Van Zee et al., 2003; Ozmen et al., 2006; Beriwal et al., 2008; Fujii et al., 2010; Boler et al., 2012; Derici et al., 2012; Scomersi et al., 2012), although in some other studies this relationship was not found or evaluated (Yu et al., 2005; Wada et al., 2006; Guray Durak et al., 2011). In a meta-analysis by Degmin et al., the size of the SLN metastasis, ECI, primary tumor size, and LVI, were defined as predicting factors of NSLNM (Degnim et al., 2003).

The relationship between the primary tumor size and NSLNM was investigated in several studies, and primary tumor size was considered to be a strong predictor of NSLN involvement. Patients with tumors larger than $20 \mathrm{~mm}$ were more likely to have NSLNM (Chu et al., 1999; Wada et al., 2006; Kapur et al., 2007; Friedman et al., 2013), although, this was not shown in a few studies (Abdessalam et al., 2001; Rahusen et al., 2001; Guray Durak et al., 2011; Eldweny et al., 2012). We found that primary tumor size is a significant predictor of NSLNM and patients with a tumor size larger than $20 \mathrm{~mm}$ were at increased risk of tumoral involvement in the remaining axillary lymph nodes.

Axillary lymph node involvement has been shown to be higher in ER/PR positive patients in some investigations (Bevilacqua et al., 2007). Van Calster et al. found that ER and HER-2/neu positive tumors have a higher likelihood of axillary lymph node involvement (Van Calster et al., 2009). We did not find any relationship between ER, PR and P53 status and HER-2/neu expression, with NSLNM. Kwon et al., investigated the association of numerous biological markers and NSLNM. They reported that biomarkers are not useful predictors of NSLNM (Kwon et al., 2011).

In this study, no significant relationship was found between PNI, size of SLN metastasis, multifocality of the primary tumor and the number of positive SLNs with NSLNM.

Some investigations have revealed that multifocality of the primary tumor is a predictor of NSLNM (Ozmen et al., 2006; Fougo et al., 2009). In the present study, the relationship between multifocality of the primary tumor and NSLNM was significant in univariate analysis, but it was not significant in multivariate analysis. The reason for this finding may be the low number of patients with multifocal tumors in our study (27 patients).

The size of the SLN metastasis had no significant relationship with NSLNM after multivariate analysis. Some investigations have demonstrated that the presence of micrometastasis in SLN was associated with lower rates of NSLNM, compared to macrometastasis (Chu et al., 1999; Van Deurzen et al., 2007; Baker et al., 2012; Mittendorf et al., 2012). Fougo et al. reported that the size of the SLN metastasis was not an independent predictor of NSLNM (Fougo et al., 2009). The small number of micrometastasis in our study population might be the reason for the differences between our results and other studies (only 14 patients had micrometastasis in SLN).

Based on the identified predictors of NSLNM in different studies, several nomograms have been developed to predict the presence of tumor in NSLNs in the axilla (Van Zee et al., 2003; Barranger et al., 2005; Kohrt et al., 2008; Pal et al., 2008; Gur et al., 2010; Koca et al., 2014). The most widely used nomogram is developed by Memorial Sloan-Kettering Cancer Center (MSKCC) (Van Zee et al., 2003). This nomogram includes primary tumor size, grade, number of positive and negative SLNs, SLN detection method, ER status, LVI, and tumor multifocality to predict NSLNM. Although the predictive accuracy of these nomograms have been validated, they are not widely used due to their complexity.

The current study had some limitations. In our institution, SLNs are not routinely evaluated with immunohistochemistry methods and this might be a reason for the low incidence of micrometastasis in our patients. In addition, this was a retrospective study and some data were not available for all patients which could have affected our results.

In conclusion, overall, in our study, predicting factors of NSLNM were age, LVI, ECI, primary tumor size, and PSLNs/TSLNs ratio. These factors should be validated in prospective studies in order to develop and validate a nomogram to predict NSLNM in Iranian patients.

\section{References}

Abdessalam SF, Zervos EE, Prasad M, et al (2001). Predictors of positive axillary lymph nodes after sentinel lymph node biopsy in breast cancer. Am J Surg, 182, 316-20.

Afsharfard A, Mozaffar M, Orang E, et al (2013). Trends in Epidemiology, Clinical and Histopathological Characteristics of Breast Cancer in Iran: Results of a 17 Year Study. Asian Pac J Cancer Prev, 14, 6905-11.

Aitken E, Osman M (2010). Factors affecting nodal status in invasive breast cancer: a retrospective analysis of 623 patients. Breast J, 16, 271-8.

Altinyollar H, Berberoglu U, Gulben K, et al (2007). The correlation of extranodal invasion with other prognostic parameters in lymph node positive breast cancer. $J$ Surg Oncol, 95, 567-71.

Alvarenga CA, Santos CCd, Alvarenga M, et al (2013). Localization of metastasis within the sentinel lymph node biopsies: a predictor of additional axillary spread of breast cancer? Rev Bras Ginecol Obstet, 35, 483-99.

Ashikaga T, Krag DN, Land SR, et al (2010). Morbidity results from the NSABP B-32 trial comparing sentinel lymph node dissection versus axillary dissection. J Surg Oncol, 102, 111-8.

Baker TA, Wadhwani N, Rajan P, et al (2012). The size of metastasis in the sentinel node is a predictor of additional non-sentinel node positivity. J Cancer Ther, 3, 712-7.

Barranger E, Coutant C, Flahault A, et al (2005). An axilla scoring system to predict non-sentinel lymph node status in breast cancer patients with sentinel lymph node involvement. Breast Cancer Res Treat, 91, 113-9.

Beriwal S, Soran A, Kocer B, et al (2008). Factors that predict the burden of axillary disease in breast cancer patients with a positive sentinel node. Am J Clin Oncol, 31, 34-8.

Bevilacqua JLB, Kattan MW, Fey JV, et al (2007). Doctor, what are my chances of having a positive sentinel node? A validated nomogram for risk estimation. J Clin Oncol, 
25, 3670-9.

Boler D, Uras C, Ince U, et al (2012). Factors predicting the nonsentinel lymph node involvement in breast cancer patients with sentinel lymph node metastases. The Breast, 21, 518-23.

Bolster MJ, Peer PG, Bult P, et al (2007). Risk factors for non-sentinel lymph node metastases in patients with breast cancer. The outcome of a multi-institutional study. Ann Surg Oncol, 14, 181-9.

Cady B (2001). Sentinel lymph node biopsy as an alternative to routine axillary lymph node dissection in breast cancer patients. J Surg Oncol, 77, 149-52.

Chu KU, Turner RR, Hansen NM, et al (1999). Do all patients with sentinel node metastasis from breast carcinoma need complete axillary node dissection? Ann Surg, 229, 536-41.

Degnim AC, Griffith KA, Sabel MS, et al (2003). Clinicopathologic features of metastasis in nonsentinel lymph nodes of breast carcinoma patients. Cancer, 98, 2307-15.

Derici S, Sevinc A, Harmancioglu O, et al (2012). Validation of three breast cancer nomograms and a new formula for predicting non-sentinel lymph node status. Asian Pac J Cancer Prev, 13, 6181-5.

Dubsky PC, Gnant MF, Taucher S, et al (2002). Young age as an independent adverse prognostic factor in premenopausal patients with breast cancer. Clin Breast Cancer, 3, 65-72.

Eldweny H, Alkhaldy K, Alsaleh N, et al (2012). Predictors of non-sentinel lymph node metastasis in breast cancer patients with positive sentinel lymph node (Pilot study). J Egypt Natl Canc Inst, 24, 23-30.

Fant JS, Grant MD, Knox SM, et al (2003). Preliminary outcome analysis in patients with breast cancer and a positive sentinel lymph node who declined axillary dissection. Ann Surg Oncol, 10, 126-30.

Farshid G, Pradhan M, Kollias J, et al (2004). A decision aid for predicting non-sentinel node involvement in women with breast cancer and at least one positive sentinel node. The Breast, 13, 494-501.

Fougo JL, Afonso M, Senra FS, et al (2009). Predictive factors for non-sentinel lymph node involvement in breast cancer patients with a positive sentinel node: should we consider sentinel node-related factors? Clin Transl Oncol, 11, 165-71.

Friedman D, Gipponi M, Murelli F, et al (2013). Predictive factors of non-sentinel lymph node involvement in patients with invasive breast cancer and sentinel node micrometastases. Anticancer Res, 33, 4509-14.

Fujii T, Yanagita Y, Fujisawa T, et al (2010). Implication of extracapsular invasion of sentinel lymph nodes in breast cancer: prediction of nonsentinel lymph node metastasis. World J Surg, 34, 544-8.

Giuliano AE, Hunt KK, Ballman KV, et al (2011). Axillary dissection vs no axillary dissection in women with invasive breast cancer and sentinel node metastasis: a randomized clinical trial. JAMA, 305, 569-75.

Goyal A, Douglas-Jones A, Newcombe R, et al (2004). Predictors of non-sentinel lymph node metastasis in breast cancer patients. Eur J Cancer, 40, 1731-7.

Guenther JM, Hansen NM, DiFronzo LA, et al (2003). Axillary dissection is not required for all patients with breast cancer and positive sentinel nodes. Arch Surg, 138, 52-6.

Gur A, Unal B, Ozbek U, et al (2010). Validation of breast cancer nomograms for predicting the non-sentinel lymph node metastases after a positive sentinel lymph node biopsy in a multi-center study. Eur J Surg Oncol, 36, 30-5.

Guray Durak M, Akansu B, Akin MM, et al (2011). Factors predicting non-sentinel lymph node involvement in sentinel node positive breast carcinoma. Turk Patoloji Derg, 27, 189-95.

Harirchi I, Karbakhsh M, Kashefi A, et al (2004). Breast cancer in Iran: results of a multi-center study. Asian Pac J Cancer Prev, 5, 24-7.

Jeruss JS, Winchester DJ, Sener SF, et al (2005). Axillary recurrence after sentinel node biopsy. Ann Surg Oncol, 12, 34-40.

Jinno H, Sakata M, Asaga S, et al (2008). Predictors to assess non-sentinel lymph node status in breast cancer patients with sentinel lymph node metastasis. Breast J, 14, 551-5.

Kapur U, Rubinas T, Ghai R, et al (2007). Prediction of nonsentinel lymph node metastasis in sentinel node-positive breast carcinoma. Ann Diagn Pathol, 11, 10-2.

Koca B, Kuru B, Ozen N, et al (2014). A breast cancer nomogram for prediction of non-sentinel node metastasis-validation of fourteen existing models. Asian Pac J Cancer Prev, 15, $1481-8$.

Kohrt HE, Olshen RA, Bermas HR, et al (2008). New models and online calculator for predicting non-sentinel lymph node status in sentinel lymph node positive breast cancer patients. BMC Cancer, $\mathbf{8}, 66$.

Kwon Y, Ro J, Kang H-S, et al (2011). Clinicopathological parameters and biological markers predicting non-sentinel node metastasis in sentinel node-positive breast cancer patients. Oncol Rep, 25, 1063-71.

Lucci A, McCall LM, Beitsch PD, et al (2007). Surgical complications associated with sentinel lymph node dissection (SLND) plus axillary lymph node dissection compared with SLND alone in the american college of surgeons oncology group trial Z0011. J Clin Oncol, 25, 3657-63.

McMasters KM, Tuttle TM, Carlson DJ, et al (2000). Sentinel lymph node biopsy for breast cancer: a suitable alternative to routine axillary dissection in multi-institutional practice when optimal technique is used. J Clin Oncol, 18, 2560-6.

Mittendorf EA, Hunt KK, Boughey JC, et al (2012). Incorporation of sentinel lymph node metastasis size into a nomogram predicting nonsentinel lymph node involvement in breast cancer patients with a positive sentinel lymph node. Ann Surg, 255, 109-15.

Mousavi SM, Montazeri A, Mohagheghi MA, et al (2007). Breast cancer in Iran: an epidemiological review. Breast $J$, 13, 383-91

Noguchi M (2008). Avoidance of axillary lymph node dissection in selected patients with node-positive breast cancer. Eur $J$ Surg Oncol, 34, 129-34.

Ozmen V, Karanlik H, Cabioglu N, et al (2006). Factors predicting the sentinel and non-sentinel lymph node metastases in breast cancer. Breast Cancer Res Treat, 95, 1-6.

Pal A, Provenzano E, Duffy S, et al (2008). A model for predicting non-sentinel lymph node metastatic disease when the sentinel lymph node is positive. Br J Surg, 95, 302-9.

Rahusen FD, Torrenga H, van Diest PJ, et al (2001). Predictive factors for metastatic involvement of nonsentinel nodes in patients with breast cancer. Arch Surg, 136, 1059-63.

Schrenk P, Rieger R, Shamiyeh A, et al (2000). Morbidity following sentinel lymph node biopsy versus axillary lymph node dissection for patients with breast carcinoma. Cancer, 88, 608-14.

Scomersi S, Torelli L, Zanconati F, et al (2012). Evaluation of a breast cancer nomogram for predicting the likelihood of additional nodal metastases in patients with a positive sentinel node biopsy. Ann Ital Chir, 83, 461-8.

Silverstein MJ, Skinner KA, Lomis TJ (2001). Predicting axillary nodal positivity in 2282 patients with breast carcinoma. World J Surg, 25, 767-72.

Singletary SE, Allred C, Ashley P, et al (2002). Revision of the American Joint Committee on Cancer staging system for breast cancer. J Clin Oncol, 20, 3628-36.

Singletary SE, Greene FL (2003). Revision of breast cancer 
staging: the 6th edition of the TNM Classification. Semin Surg Oncol, 21, 53-9

Stitzenberg KB, Meyer AA, Stern SL, et al (2003). Extracapsular extension of the sentinel lymph node metastasis: a predictor of nonsentinel node tumor burden. Ann Surg, 237, 607-12.

Turner RR, Chu KU, Qi K, et al (2000). Pathologic features associated with nonsentinel lymph node metastases in patients with metastatic breast carcinoma in a sentinel lymph node. Cancer, 89, 574-81.

Van Calster B, Bempt IV, Drijkoningen M, et al (2009). Axillary lymph node status of operable breast cancers by combined steroid receptor and HER-2 status: triple positive tumours are more likely lymph node positive. Breast Cancer Res Treat, 113, 181-7.

Van Deurzen C, Van Hillegersberg R, Hobbelink M, et al (2007). Predictive value of tumor load in breast cancer sentinel lymph nodes for second echelon lymph node metastases. Cell Oncol, 29, 497-505.

Van la Parra R, Peer P, Ernst M, et al (2011). Meta-analysis of predictive factors for non-sentinel lymph node metastases in breast cancer patients with a positive SLN. Eur J Surg Oncol, 37, 290-9.

Van Zee KJ, Manasseh D-ME, Bevilacqua JL, et al (2003). A nomogram for predicting the likelihood of additional nodal metastases in breast cancer patients with a positive sentinel node biopsy. Ann Surg Oncol, 10, 1140-51.

Viale G, Maiorano E, Pruneri G, et al (2005). Predicting the risk for additional axillary metastases in patients with breast carcinoma and positive sentinel lymph node biopsy. Ann Surg, 241, 319-25.

Wada N, Imoto S, Yamauchi C, et al (2006). Predictors of tumour involvement in remaining axillary lymph nodes of breast cancer patients with positive sentinel lymph node. Eur $J$ Surg Oncol, 32, 29-33.

Yu J, Hsu GC, Hsieh CB, et al (2005). Prediction of metastasis to non-sentinel nodes by sentinel node status and primary tumor characteristics in primary breast cancer in Taiwan. World J Surg, 29, 813-8. 